\title{
Estimation of Heat Flux in Two-Dimensional Nonhomogeneous Parabolic Equation Based on a Sufficient Descent Levenberg-Marquard Algorithm
}

\author{
Xinfu Pang $\mathbb{D}^{1},{ }^{1}$ Yang Yu, ${ }^{2,3}$ Haibo Li, ${ }^{1}$ Yuan Wang, ${ }^{4}$ and Jinhui Zhao ${ }^{5}$ \\ ${ }^{1}$ School of Automation, Shenyang Institute of Engineering, Shenyang 110136, China \\ ${ }^{2}$ School of Automation, Shenyang Aerospace University, Shenyang 110136, China \\ ${ }^{3}$ School of Mechatronic Engineering and Automation, Shanghai University, Shanghai 200072, China \\ ${ }^{4}$ School of Science and Engineering, The Chinese University of Hong Kong, Shenzhen 518172, China \\ ${ }^{5}$ College of Electrical Engineering, Zhejiang University of Water Resources and Electric Power, Hangzhou 310018, China \\ Correspondence should be addressed to Xinfu Pang; pangxf@sie.edu.cn
}

Received 8 November 2020; Revised 27 March 2021; Accepted 21 May 2021; Published 28 May 2021

Academic Editor: Basil Papadopoulos

Copyright (c) 2021 Xinfu Pang et al. This is an open access article distributed under the Creative Commons Attribution License, which permits unrestricted use, distribution, and reproduction in any medium, provided the original work is properly cited.

\begin{abstract}
The main work of this paper focuses on identifying the heat flux in inverse problem of two-dimensional nonhomogeneous parabolic equation, which has wide application in the industrial field such as steel-making and continuous casting. Firstly, the existence of the weak solution of the inverse problem is discussed. With the help of forward solution and dual equation, this paper proves the Lipchitz continuity of the cost function and derives the Lipchitz constant. Furthermore, in order to accelerate the convergence rate and reduce the running time, this paper presents a sufficient descent Levenberg-Marquard algorithm with adaptive parameter (SD-LMAP) to solve this inverse problem. At last, compared with other methods, the results of simulation experiment show that this algorithm can obviously reduce the running time and iterative number.
\end{abstract}

\section{Introduction}

The most steel production is completed by the continuous casting machine. To guarantee the quality of casting billet, the spray cooling control system $[1,2]$ based on the heat transfer model is considered. Hence, the accurate heat transfer model counts a great deal for the production of continuous casting. Boundary conditions are essential to the accurate heat transfer model. Therefore, it is crucial to correct boundary conditions along the caster machine during the casting operations. Heat flux at the metal/cooling interface is an important one among these boundary conditions $[3,4]$. The identification of heat flux of the heat transfer model in continuous casting is a kind of inverse problems of the parabolic equation. The inverse problems of the parabolic equation are encountered in many applications, in particular, in heat transfer [5, 6], filtration [7], and material science $[8,9]$. We only consider the inverse problem in continuous casting.

Recently, the inverse problems of the parabolic equation have been considered by many authors. Wang et al. [10] used a regularized optimization method for identifying the spacedependent source in a parabolic equation. Ruan et al. [11] investigated an inverse source problem for the time-fractional diffusion equation, which is formulated into an optimization problem, and a semismooth Newton (SSN) algorithm is developed to solve it. Chen et al. [12] applied an inverse algorithm based on the conjugate gradient method and the discrepancy principle to solve the inverse hyperbolic heat conduction problem. Zhao and Banda [13] deal with an inverse problem of determining the diffusion coefficient, spacewise dependent source term, and initial value simultaneously for the one-dimensional heat equation. Beck and Woodbury [14] estimated the time and/or space dependence 
of the surface heat flux or temperature utilizing interior temperature measurements at discrete times and/or locations. Lee [15] demonstrated the performance and efficiency of the repulsive particle swarm optimization (RPSO) method for solving the inverse heat conduction problem. Cui et al. $[16,17]$ developed an improved Levenberg-Marquardt (L$\mathrm{M})$ algorithm for solving inverse problems. Oliveira et al [18] used the Levenberg-Marquardt technique to determine the interfacial heat transfer coefficient.

Our previous works $[19,20]$ focused on the identification of the heat transfer coefficients of the heat transfer model by the L-M algorithm. Based on the above works, this paper considers the inverse problem of two-dimensional nonhomogeneous parabolic equation associated with the continuous casting. This problem is described as a PDE optimization problem. The adjoint approach is used to prove the Lipchitz continuous of the gradient of cost function in PDE optimization problem with the two-dimensional nonhomogeneous parabolic equation. Under the condition of Lipchitz continuous, this paper presents a sufficient descent Levenberg-Marquard with adaptive parameter (SDLMAP) algorithm to solve this problem. And, the simulation experiment is used to illustrate the validity of this algorithm.

The construction of paper is organized as follows. In Section 2, the heat transfer model of continuous casting is given. And, we prove the Lipchitz continuous of the gradient of cost function in Section 3. In Section 4, the sufficient descent Levenberg-Marquard (SD-LMAP) algorithm with an adaptive parameter is presented. In Section 5, the simulation experiments are given to illustrate the validity of the proposed method.

\section{Heat Transfer Model for Continuous Casting and the Description of the Problem}

2.1. Heat Transfer Mathematical Model for Continuous Casting. The pouring process of continuous casting is given in Figure 1, and the solidification of the slab can be described by the heat transfer model. The unsteady state heat transfer mathematical model based on some assumptions can be used to predict the temperature distribution during the process of casting solidification. So, we assume as follows: (1) in the process of the continuous casting, convective heat transfer is equal to the thermal conduction, (2) the mold is considered uniform and the cooling temperature equal to the water-cooling temperature $\left(u_{w}\right)$, and (3) the meniscus surface is considered to be flat. Based on the above assumptions, the two-dimensional heat transfer model [1] is given by

$$
\rho c \frac{\partial u}{\partial t}=k\left(\frac{\partial^{2} u}{\partial x^{2}}+\frac{\partial^{2} u}{\partial y^{2}}\right)+Q(x, y, t), \quad(x, y, t) \in \Omega,
$$

where $\Omega:=\left\{(x, y, t) \in \Re^{3}: x \in[0, l], y \in[0, l], t \in(0, T]\right.$, $T>0\}, l$ is the width of billet (the width is equal to the thickness for billet), $T$ is the casting time $(s), \rho$ is density $\left(\mathrm{kg} / \mathrm{m}^{3}\right), c$ is specific heat $(\mathrm{J} /(\mathrm{kg} \cdot \mathrm{K})), k$ is thermal conductivity $(\mathrm{W} /(\mathrm{m} \cdot \mathrm{K})), u$ is temperature $(K), t$ is time $(s)$,

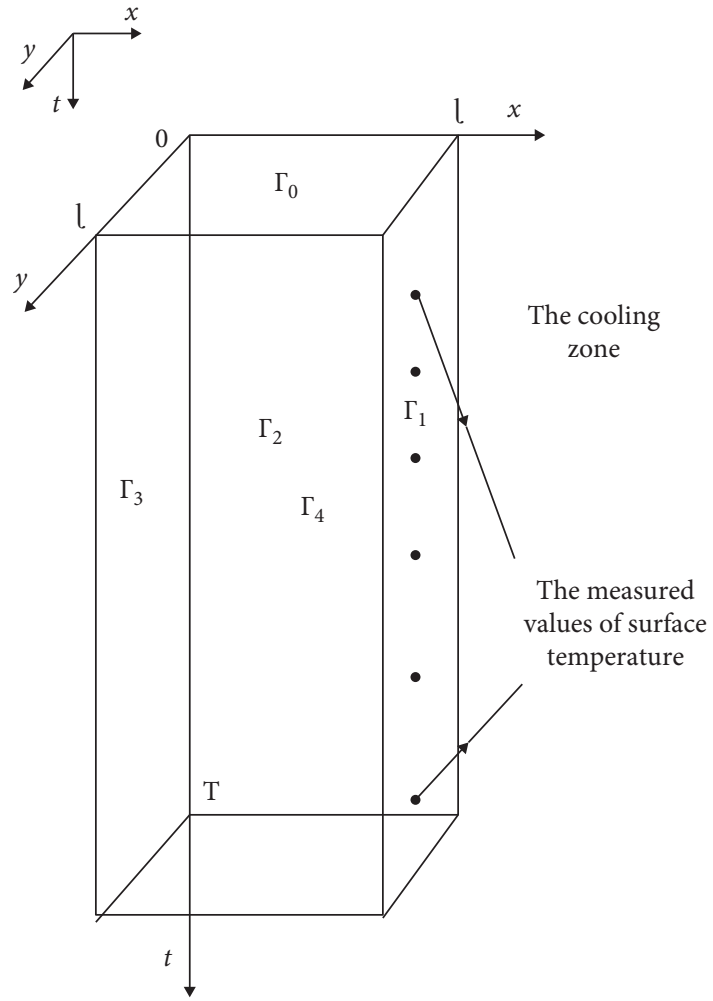

FIgURE 1: The pouring process of continuous casting.

and $Q$ is the term associated to the internal heat generation because of the phase change.

The boundary condition at beginning (on $\Gamma_{0}$ ) is

$$
\left.u_{\text {begin }}(x, y, t)\right|_{t=0}=u_{\text {cast }}, \quad x \in[0, l], y \in[0, l],
$$

where $u_{\text {cast }}$ is the pouring temperature $(K)$, which is considered as a constant.

On the boundary $\Gamma_{1}(x=1)$ and $\Gamma_{2}(y=1)$, the spray cooling method is used to solidify the billet, and these boundary conditions can be written as

$$
\begin{array}{ll}
-\left.k \frac{\partial u(x, y, t)}{\partial x}\right|_{x=l}=q(y, t), & y \in[0, l], t \in(0, T], \\
-\left.k \frac{\partial u(x, y, t)}{\partial y}\right|_{y=l}=q(x, t), \quad x \in[0, l], t \in(0, T],
\end{array}
$$

where $q(x, t)=\bar{\gamma} *\left(u(x, l, t)-u_{w}\right)$ is heat flux on $\Gamma_{2}=\{x \in(0, l), t \in(0, T)\}$, which is crucial to the heat transfer model. $\bar{\gamma}$ is heat transfer coefficient and $u_{w}$ is the temperature of cooling water. However, the interface heat flux of the billet is difficult to be measured, so this paper considers to identify this parameter. Similarly, $q(y, t)=\bar{\gamma} *\left(u(l, y, t)-u_{w}\right)$ is the heat flux on $\Gamma_{1}=\{y \in(0, l), t \in(0, T)\}$. The cooling intensity on the surface of width is the same as the thickness for billet.

Because of the symmetrical heat flux at the center of the slab, we can consider the $1 / 4$ of steel billet, and the boundary conditions on $\Gamma_{3}(x=0)$ and $\Gamma_{4}(y=0)$ are given in the following: 


$$
\begin{aligned}
& -\left.k \frac{\partial u(x, y, t)}{\partial x}\right|_{x=0}=0, \quad y \in[0, l], t \in(0, T] \\
& -\left.k \frac{\partial u(x, y, t)}{\partial y}\right|_{y=0}=0, \quad x \in[0, l], t \in(0, T] .
\end{aligned}
$$

2.2. The Description of the Problem. In the productive process of continuous casting, the surface temperature of billet $(x=l$ or $y=l$ ) can be measured [3]. We can use these data to identify the heat flux $q(\cdot, t)$. We define $a=(k / \rho c)$ and $h(\cdot, t)=q(\cdot, t) /(\rho c)$, and the inverse problem can be described by the following PDE optimization problem:

$$
\begin{aligned}
J(h) & =J_{1}(h)+J_{2}(h), \\
\text { s.t. } u_{t} & =a\left(u_{x x}+u_{y y}\right)+Q(x, y, t),(x, y, t) \in \Omega, \\
\left.u(x, y, t)\right|_{t=0} & =u_{0}, \quad x \in[0, l], y \in[0, l], \\
\left.a u_{y}(x, y, t)\right|_{y=l} & =-h(x, t),\left.a u_{y}(x, y, t)\right|_{y=0} \\
& =0, t \in(0, T], x \in[0, l], \\
\left.a u_{x}(x, y, t)\right|_{x=l} & =-h(y, t),\left.a u_{x}(x, y, t)\right|_{x=0} \\
& =0, t \in(0, T], y \in[0, l],
\end{aligned}
$$

where ${ }_{l}^{l} J_{1}(h)=\int_{0}^{l} \int_{0}^{T}\left\|u_{c}(l, y, t ; h)-u_{e}^{\delta}(l, y, t)\right\|^{2} \mathrm{~d} y \mathrm{~d} t$, $J_{2}(h)=\int_{0}^{l} \int_{0}^{T}\left\|u_{c}(x, l, t ; h)-u_{e}^{\delta}(x, l, t)\right\|^{2} \mathrm{~d} x \mathrm{~d} t, \quad u_{c}(l, y, t ; h)$ and $u_{c}(x, l, t ; h)$ are calculated values of the surface temperature on $\Gamma_{1}$ and $\Gamma_{2}$, respectively, $u_{e}^{\delta}(l, y, t)$ and $u_{e}^{\delta}(x, l, t)$ are measured values of the surface temperature on $\Gamma_{1}$ and $\Gamma_{2}$, respectively, $\delta$ is the error level, and $u_{0}$ is the initial temperature, where we define $u_{0}=u_{\text {begin }}-u_{\text {cast }}=0$.

Remark 1. Because the cooling intensity on the surface of width is the same as thickness for billet, as long as the heat flux on width side is estimated, the heat flux on the other side can be known. In the process of measurement, we only need to measure the surface temperature on one side.

\section{The Quasi-Solution of the Inverse Problem and Lipchitz Continuity of the Gradient of the Cost Function}

3.1. The Quasi-Solution Approach Based on Weak Solution. We denote the set of admissible unknown heat flux $h(\cdot, t)$ by $H(\Omega)$, which satisfies the following equation:

$$
h(\cdot, t) \in H(\Omega), \quad \forall t \in(0, T], x \in[0, l], y \in[0, l] .
$$

The weak solution of the forward problem (7) will be defined to be the function $u \in \dot{V}^{1,0}(\Omega)$, which satisfies the following integral identity:

$$
\iiint_{\Omega}\left(-u v_{t}+a v_{x} u_{x}+a v_{y} u_{y}\right) \mathrm{d} x \mathrm{~d} y \mathrm{~d} t=\iiint_{\Omega} Q(x, y, t) v \mathrm{~d} x \mathrm{~d} y \mathrm{~d} t, \quad \forall v \in H(\Omega),
$$

where $v(x, y, T)=0$ for $x \in[0, l]$ and $y \in[0, l]$ and $v(l, y, t)=0$ and $v(x, l, t)=0$ for $t \in(0, T]$. Obviously, under the above conditions with regards to the given data, the existence and uniqueness of this solution in $u \in \dot{V}^{1,0}(\Omega)$ can be found in $[5,21]$.

Let us denote the solution of problem (7) by $u^{*}(x, y, t ; h), \quad$ corresponding to a given $h(\cdot, t) \in H(\Omega), \forall t \in(0, T], x \in[0, l], y \in[0, l] . \quad$ If this function satisfies the measured data $u_{e}^{\delta}(x, l, t)$ or $u_{e}^{\delta}(l, y, t)$, then it should satisfy the nonlinear functional equation:

$$
u^{*}\left(\left.(x, y, t ; h)\right|_{x=l}=u_{e}^{\delta}(l, y, t), \quad t \in(0, T], y \in[0, l],\right.
$$

$$
\text { or }
$$

$$
\left.u^{*}(x, y, t ; h)\right|_{y=l}=u_{e}^{\delta}(x, l, t), \quad t \in(0, T], x \in[0, l] .
$$

In practice, the measured data $u_{e}^{\delta}(x, 0, t)$ is usually given by the data with error level $\delta$. The exact fulfillment of condition (10) may be not possible. According to this reason, a quasi-solution of the inverse problem (7) is defined as a solution of the following minimization problem:

$$
J\left(h^{*}\right)=\inf J(h),
$$

where $J(h)$ is the cost functional, which is defined by equation (7). 
We assume that $h, h+\Delta h \in H$, and the variation of the cost function $\Delta J$ can be given in the following:

$$
\begin{aligned}
\Delta J(h)= & \Delta J_{1}(h)+\Delta J_{2}(h) \\
= & 2 \int_{0}^{T} \int_{0}^{l}\left[u_{c}(x, l, t ; h)-u_{e}^{\delta}\right] \Delta u(x, l, t ; \Delta h) \mathrm{d} x \mathrm{~d} t+\int_{0}^{T} \int_{0}^{l}[\Delta u(x, l, t ; \Delta h)]^{2} \mathrm{~d} x \mathrm{~d} t \\
& +2 \int_{0}^{T} \int_{0}^{l}\left[u_{c}(l, y, t ; h)-u_{e}^{\delta}\right] \Delta u(l, y, t ; \Delta h) \mathrm{d} y \mathrm{~d} t+\int_{0}^{T} \int_{0}^{l}[\Delta u(l, y, t ; \Delta h)]^{2} \mathrm{~d} y \mathrm{~d} t,
\end{aligned}
$$

where $\quad \Delta u(x, y, t ; h)=u(x, y, t ; h+\Delta h)-u(x, y, t ; h) \epsilon$ $H(\Omega)$. Obviously, $\Delta u=\Delta u(x, y, t ; \Delta h)$ is the solution of the following parabolic problem:

$$
\begin{aligned}
\Delta u_{t} & =a\left(\Delta u_{x x}+\Delta u_{y y}\right), \quad(x, y, t) \in \Omega, \\
\Delta u(x, y, 0) & =0, \quad x \in[0, l], y \in[0, l], \\
\left.a \Delta u_{y}(x, y, t)\right|_{y=l} & =-\Delta h(x, t), \\
\left.a \Delta u_{y}(x, y, t)\right|_{y=0} & =0, \quad t \in(0, T], x \in[0, l], \\
\left.a \Delta u_{x}(x, y, t)\right|_{x=l} & =-\Delta h(y, t), \\
\left.a \Delta u_{x}(x, y, t)\right|_{x=0} & =0, \quad t \in(0, T], y \in[0, l] .
\end{aligned}
$$

Lemma 1. If $u=u(x, y, t ; h) \in H(\Omega)$ is the corresponding solution of the direct problem (7) and $\varphi(x, y, t ; h) \in H(\Omega)$ is the solution of the adjoint problem,

$$
\begin{aligned}
\varphi_{t} & =-a\left(\varphi_{x x}+\varphi_{y y}\right), \quad(x, y, t) \in \Omega, \\
\varphi(x, y, T) & =0, \quad x \in[0, l], y \in[0, l], \\
-\left.a \varphi_{y}(x, y, t)\right|_{y=0} & =0, \\
-\left.a \varphi_{y}(x, y, t)\right|_{y=l} & =2\left[u_{c}(x, l, t ; h)-u_{e}^{\delta}(x, l, t)\right], \quad t \in[0, T], x \in[0, l], \\
-\left.a \varphi_{x}(x, y, t)\right|_{x=0} & =0, \\
-\left.a \varphi_{x}(x, y, t)\right|_{x=l} & =2\left[u_{c}(l, y, t ; h)-u_{e}^{\delta}(l, y, t)\right], \quad t \in[0, T], y \in[0, l] .
\end{aligned}
$$

For all $\Delta h$, we have the following identity:

$$
\begin{aligned}
& 2 \int_{0}^{T} \int_{0}^{l}\left[u_{c}(x, l, t ; h)-u_{e}^{\delta}(x, l, t)\right] \Delta u(x, l, t) \mathrm{d} x \mathrm{~d} t=\int_{0}^{T} \int_{0}^{l} \varphi(x, l, t) \Delta h(x, t) \mathrm{d} x \mathrm{~d} t, \\
& 2 \int_{0}^{T} \int_{0}^{l}\left[u_{c}(l, y, t ; h)-u_{e}^{\delta}(l, y, t)\right] \Delta u(l, y, t) \mathrm{d} y \mathrm{~d} t=\int_{0}^{T} \int_{0}^{l} \varphi(l, y, t) \Delta h(y, t) \mathrm{d} y \mathrm{~d} t .
\end{aligned}
$$

Proof. Multiplying both sides of parabolic (14) by $\varphi(x, y, t)$ and integrating on $\Omega$, based on the initial and boundary conditions, we have the following 


$$
\begin{aligned}
& \iiint_{\Omega}\left[\Delta u_{t}-a\left(\Delta u_{x x}+\Delta u_{y y}\right)\right] \varphi(x, y, t) \mathrm{d} x \mathrm{~d} y \mathrm{~d} t \\
& =-\int_{0}^{T} \int_{0}^{l} \int_{0}^{l} \varphi_{t}(x, y, t) \Delta u \mathrm{~d} x \mathrm{~d} y \mathrm{~d} t-\int_{0}^{T} \int_{0}^{l} \int_{0}^{l} a \varphi_{x x}(x, y, t) \Delta u \mathrm{~d} x \mathrm{~d} y \mathrm{~d} t-\int_{0}^{T} \int_{0}^{l} \int_{0}^{l} a \varphi_{y y}(x, y, t) \Delta u \mathrm{~d} x \mathrm{~d} y \mathrm{~d} t \\
& \quad-\left.\int_{0}^{T} \int_{0}^{l} a \varphi(x, y, t) \Delta u_{x}\right|_{x=0} ^{x=l} \mathrm{~d} y \mathrm{~d} t+\left.\int_{0}^{T} \int_{0}^{l} a \varphi_{x}(x, y, t) \Delta u\right|_{x=0} ^{x=l} \mathrm{~d} y \mathrm{~d} t \\
& \quad-\left.\int_{0}^{T} \int_{0}^{l} a \varphi(x, y, t) \Delta u_{y}\right|_{y=0} ^{y=l} \mathrm{~d} y \mathrm{~d} t+\left.\int_{0}^{T} \int_{0}^{l} a \varphi_{y}(x, y, t) \Delta u\right|_{y=0} ^{y=l} \mathrm{~d} y \mathrm{~d} t .
\end{aligned}
$$

According to equation (15), we have

$$
\begin{aligned}
& \iiint_{\Omega}\left[\Delta u_{t}-a\left(\Delta u_{x x}+\Delta u_{y y}\right)\right] \varphi(x, y, t) \mathrm{d} x \mathrm{~d} y \mathrm{~d} t \\
& =-\left.\int_{0}^{T} \int_{0}^{l} a \varphi(x, y, t) \Delta u_{x}\right|_{x=0} ^{x=l} \mathrm{~d} y \mathrm{~d} t+\left.\int_{0}^{T} \int_{0}^{l} a \varphi_{x}(x, y, t) \Delta u\right|_{x=0} ^{x=l} \mathrm{~d} y \mathrm{~d} t \\
& \quad-\left.\int_{0}^{T} \int_{0}^{l} a \varphi(x, y, t) \Delta u_{y}\right|_{y=0} ^{y=l} \mathrm{~d} y \mathrm{~d} t+\left.\int_{0}^{T} \int_{0}^{l} a \varphi_{y}(x, y, t) \Delta u\right|_{y=0} ^{y=l} \mathrm{~d} y \mathrm{~d} t=0 .
\end{aligned}
$$

Using the conditions of equations (14) and (15), we have

$$
\begin{aligned}
& \int_{0}^{T} \int_{0}^{l} 2\left[u_{c}(x, l, t ; h)-u_{e}^{\delta}(x, l, t)\right] \mathrm{d} x \mathrm{~d} t \\
& \quad+\int_{0}^{T} \int_{0}^{l} 2\left[u_{c}(l, y, t ; h)-u_{e}^{\delta}(l, y, t)\right] \mathrm{d} y \mathrm{~d} t \\
& =\int_{0}^{T} \int_{0}^{l} \varphi(l, y, t) \Delta h(y, t) \mathrm{d} y \mathrm{~d} t \\
& \quad+\int_{0}^{T} \int_{0}^{l} \varphi(x, l, t) \Delta h(x, t) \mathrm{d} x \mathrm{~d} t .
\end{aligned}
$$

So, Lemma 1 can be proved.

Lemma 2. Let $h \in H(\Omega)$; then, for the solution $\Delta u:=\Delta u(x, y, t ; \Delta h) \in \dot{V}^{1,0}(\Omega)$ of the parabolic problem (14), there exists constants $\varepsilon_{1}\left(0<\varepsilon_{1}<(2 / l)\right)$ and $\varepsilon_{1}\left(0<\varepsilon_{2}<(2 / l)\right)$, which satisfies $\bar{c}_{1}=\left(l / \varepsilon_{1}\left(2-\varepsilon_{1} l\right)\right)$ and $\bar{c}_{2}=\left(l / \varepsilon_{2}\left(2-\varepsilon_{2} l\right)\right)$, and the following equation can be obtained for a given $\Delta h(\cdot, t)$ :

$$
\begin{gathered}
\|\Delta u(x, l, t)\|^{2} \leq \bar{c}_{1}\|\Delta h(x, t)\|^{2}, \\
\|\Delta u(l, y, t)\|^{2} \leq \bar{c}_{2}\|\Delta h(y, t)\|^{2},
\end{gathered}
$$

where $\|\Delta u(x, l, t)\|^{2}=\int_{0}^{T} \int_{0}^{l}[\Delta u(x, l, t)]^{2} d x d t, \quad \| \Delta u(l, y$, $t)\left\|^{2}=\int_{0}^{T} \int_{0}^{l}[\Delta u(l, y, t)]^{2} d y d t, \quad\right\| \Delta h(x, t) \|^{2}=\int_{0}^{T} \int_{0}^{l}[\Delta h(x$, $t)]^{2} d x d t$, and $\|\Delta h(y, t)\|^{2}=\int_{0}^{T} \int_{0}^{l}[\Delta h(y, t)]^{2} d y d t$.

Proof. If we multiply both sides of partial differential equation (14) by $\Delta u(x, y, t)$ and also simultaneously in time and space integration, we can obtain the following equation:

$$
\iiint_{\Omega}\left[\Delta u_{t}-a \Delta u_{x x}-a \Delta u_{y y}\right] \Delta u \mathrm{~d} x \mathrm{~d} y \mathrm{~d} t=0 .
$$

It is easy to know that the following equation holds:

$$
\begin{aligned}
\Delta u_{t} \Delta u & =\frac{1}{2}\left((\Delta u)^{2}\right)_{t} \\
\Delta u_{x x} \Delta u & =\left(\Delta u_{x} \Delta u\right)_{x}-\left(\Delta u_{x}\right)^{2} .
\end{aligned}
$$

Hence, we have the following equations: 


$$
\begin{aligned}
\iiint_{\Omega} \Delta u_{t} \Delta u \mathrm{~d} x \mathrm{~d} y \mathrm{~d} t & =\frac{1}{2} \iiint\left((\Delta u)^{2}\right)_{t} \mathrm{~d} x \mathrm{~d} y \mathrm{~d} t=\frac{1}{2} \int_{0}^{l} \int_{0}^{l}(\Delta u(x, y, T))^{2} \mathrm{~d} x \mathrm{~d} y, \\
a \iiint_{\Omega} \Delta u_{x x} \Delta u \mathrm{~d} x \mathrm{~d} y \mathrm{~d} t & =a \iiint_{\Omega}\left(\Delta u_{x} \Delta u\right)_{x} \mathrm{~d} x \mathrm{~d} y \mathrm{~d} t-a \iiint_{\Omega}\left(\Delta u_{x}\right)^{2} \mathrm{~d} x \mathrm{~d} y \mathrm{~d} t \\
& =\left.a \int_{0}^{l} \int_{0}^{T} \Delta u_{x} \Delta u\right|_{x=0} ^{x=l} \mathrm{~d} y \mathrm{~d} t-a \iiint_{\Omega}\left(\Delta u_{x}\right)^{2} \mathrm{~d} x \mathrm{~d} y \mathrm{~d} t \\
& =a \int_{0}^{T} \int_{0}^{l} \Delta h(y, t) \Delta u(l, y, t) \mathrm{d} y \mathrm{~d} t-a \iiint_{\Omega}\left(\Delta u_{x}\right)^{2} \mathrm{~d} x \mathrm{~d} y \mathrm{~d} t .
\end{aligned}
$$

Similarly, we also have

$$
\begin{aligned}
a \iiint_{\Omega} \Delta u_{y y} \Delta u \mathrm{~d} x \mathrm{~d} y \mathrm{~d} t & =a \iiint_{\Omega}\left(\Delta u_{y} \Delta u\right)_{y} \mathrm{~d} x \mathrm{~d} y \mathrm{~d} t-a \iiint_{\Omega}\left(\Delta u_{y}\right)^{2} \mathrm{~d} x \mathrm{~d} y \mathrm{~d} t \\
& =\left.a \int_{0}^{l} \int_{0}^{T} \Delta u_{y} \Delta u\right|_{y=0} ^{y=l} \mathrm{~d} x \mathrm{~d} t-a \iiint_{\Omega}\left(\Delta u_{y}\right)^{2} \mathrm{~d} x \mathrm{~d} y \mathrm{~d} t \\
& =a \int_{0}^{T} \int_{0}^{l} \Delta h(x, t) \Delta u(x, l, t) \mathrm{d} y \mathrm{~d} t-a \iiint_{\Omega}\left(\Delta u_{y}\right)^{2} \mathrm{~d} x \mathrm{~d} y \mathrm{~d} t .
\end{aligned}
$$

According to the boundary condition of equation (14), we have

$$
\begin{aligned}
& \iiint_{\Omega}\left[\Delta u_{t}-a \Delta u_{x x}-a \Delta u_{y y}\right] \Delta u \mathrm{~d} x \mathrm{~d} y \mathrm{~d} t \\
& =\iiint_{\Omega} \Delta u_{t} \Delta u \mathrm{~d} x \mathrm{~d} y \mathrm{~d} t-a \iiint_{\Omega} \Delta u_{x x} \Delta u \mathrm{~d} x \mathrm{~d} y \mathrm{~d} t-a \iiint_{\Omega} \Delta u_{y y} \Delta u \mathrm{~d} x \mathrm{~d} y \mathrm{~d} t \\
& =\frac{1}{2} \int_{0}^{l} \int_{0}^{l}(\Delta u(x, y, T))^{2} \mathrm{~d} x \mathrm{~d} y+a \iiint_{\Omega}\left[\left(\Delta u_{x}\right)^{2}+\left(\Delta u_{y}\right)^{2}\right] \mathrm{d} x \mathrm{~d} y \mathrm{~d} t \\
& \quad-a \int_{0}^{T} \int_{0}^{l} \Delta h(x, t) \Delta u(x, l, t) \mathrm{d} x \mathrm{~d} t-a \int_{0}^{T} \int_{0}^{l} \Delta h(y, t) \Delta u(l, y, t) \mathrm{d} x \mathrm{~d} t=0 .
\end{aligned}
$$

So, we have

$$
\begin{aligned}
& \frac{1}{2} \int_{0}^{l} \int_{0}^{l}(\Delta u(x, y, T))^{2} \mathrm{~d} x \mathrm{~d} y+a \iiint_{\Omega}\left[\left(\Delta u_{x}\right)^{2}+\left(\Delta u_{y}\right)^{2}\right] \mathrm{d} x \mathrm{~d} y \mathrm{~d} t \\
= & a \int_{0}^{T} \int_{0}^{l} \Delta h(x, t) \Delta u(x, l, t) \mathrm{d} x \mathrm{~d} t+a \int_{0}^{T} \int_{0}^{l} \Delta h(y, t) \Delta u(l, y, t) \mathrm{d} x \mathrm{~d} t .
\end{aligned}
$$

According to the $\mathcal{E}$-inequality,

$$
\begin{aligned}
\int_{0}^{T} \int_{0}^{l} \Delta h(x, t) \Delta u(x, l, t) \mathrm{d} x \mathrm{~d} t \leq & \frac{1}{2 \varepsilon_{1}} \int_{0}^{T} \int_{0}^{l}|\Delta h(x, t)|^{2} \mathrm{~d} x \mathrm{~d} t \\
& +\frac{\varepsilon_{1}}{2} \int_{0}^{T} \int_{0}^{l}|\Delta u(x, l, t)|^{2} \mathrm{~d} x \mathrm{~d} t .
\end{aligned}
$$$$
\alpha \beta \leq \varepsilon \frac{\alpha^{2}}{2}+\frac{\beta^{2}}{2 \varepsilon}, \quad \forall \alpha, \beta \in R, \forall \varepsilon>0 .
$$

The first item on the right side of equation (26) can be written as 
Similarly, the second item on the right side of equation (26) can be shown in the following:

$$
\int_{0}^{T} \int_{0}^{l} \Delta h(y, t) \Delta u(l, y, t) \mathrm{d} y \mathrm{~d} t \leq \frac{1}{2 \varepsilon_{2}} \int_{0}^{T} \int_{0}^{l}|\Delta h(y, t)|^{2} \mathrm{~d} y \mathrm{~d} t+\frac{\varepsilon_{2}}{2} \int_{0}^{T} \int_{0}^{l}|\Delta u(l, y, t)|^{2} \mathrm{~d} x \mathrm{~d} t .
$$

Furthermore, we estimate $\iiint_{\Omega}\left[\left(\Delta u_{x}\right)^{2}+\left(\Delta u_{y}\right)^{2}\right]$ $\mathrm{d} x \mathrm{~d} y \mathrm{~d} t$ by applying Cauchy inequality:

$$
\begin{aligned}
& {[\Delta u(l, y, t)-\Delta u(0, y, t)]^{2}=\left[\int_{0}^{l} \Delta u_{\zeta}(\zeta, y, t) \mathrm{d} \zeta\right]^{2} \leq l \int_{0}^{l}\left(\Delta u_{x}\right)^{2} \mathrm{~d} x, \quad x \in(0, l),} \\
& {[\Delta u(x, l, t)-\Delta u(x, 0, t)]^{2}=\left[\int_{0}^{l} \Delta u_{\zeta}(x, \zeta, t) \mathrm{d} \zeta\right]^{2} \leq l \int_{0}^{l}\left(\Delta u_{y}\right)^{2} \mathrm{~d} y, \quad y \in(0, l) .}
\end{aligned}
$$

Now, we integrate both sides of inequality (30) on $\Omega$, and we can obtain

$$
\iiint_{\Omega}[\Delta u(l, y, t)]^{2} \mathrm{~d} x \mathrm{~d} y \mathrm{~d} t \leq l^{2} \iiint_{\Omega}\left(\Delta u_{x}\right)^{2} \mathrm{~d} x \mathrm{~d} y \mathrm{~d} t .
$$

Similarly, we have

$$
\iiint_{\Omega}[\Delta u(x, l, t)]^{2} \mathrm{~d} x \mathrm{~d} y \mathrm{~d} t \leq l^{2} \iiint_{\Omega}\left(\Delta u_{y}\right)^{2} \mathrm{~d} x \mathrm{~d} t
$$

And, we use these inequalities to estimate the left-hand side of inequality (26), and we can obtain the following inequality:

$$
\begin{aligned}
\frac{1}{2} \int_{0}^{l} \int_{0}^{l}[\Delta u(x, y, T)]^{2} \mathrm{~d} x \mathrm{~d} y & +\frac{a}{l} \int_{0}^{l} \int_{0}^{T}[\Delta u(l, y, t)]^{2} \mathrm{~d} x \mathrm{~d} y \mathrm{~d} t \\
& +\frac{a}{l} \int_{0}^{l} \int_{0}^{T}[\Delta u(x, l, t)]^{2} \mathrm{~d} x \mathrm{~d} y \mathrm{~d} t \\
& \leq \frac{a}{2 \varepsilon_{1}} \int_{0}^{T} \int_{0}^{l}|\Delta h(x, t)|^{2} \mathrm{~d} x \mathrm{~d} t+a \frac{\varepsilon_{1}}{2} \int_{0}^{T} \int_{0}^{l}|\Delta u(x, l, t)|^{2} \mathrm{~d} x \mathrm{~d} t \\
& +\frac{a}{2 \varepsilon_{2}} \int_{0}^{T} \int_{0}^{l}|\Delta h(y, t)|^{2} \mathrm{~d} y \mathrm{~d} t+a \frac{\varepsilon_{2}}{2} \int_{0}^{T} \int_{0}^{l}|\Delta u(l, y, t)|^{2} \mathrm{~d} y \mathrm{~d} t .
\end{aligned}
$$

So, we have

$$
\begin{aligned}
\left(\frac{a}{l}-\frac{\varepsilon_{2}}{2} a\right) \int_{0}^{l} \int_{0}^{T}[\Delta u(l, y, t)]^{2} \mathrm{~d} x \mathrm{~d} y \mathrm{~d} t & +\left(\frac{a}{l}-\frac{\varepsilon_{1}}{2} a\right) \int_{0}^{l} \int_{0}^{T}[\Delta u(x, l, t)]^{2} \mathrm{~d} x \mathrm{~d} y \mathrm{~d} t \\
& \leq \frac{a}{2 \varepsilon_{1}} \int_{0}^{T} \int_{0}^{l}|\Delta h(x, t)|^{2} \mathrm{~d} x \mathrm{~d} t+\frac{a}{2 \varepsilon_{2}} \int_{0}^{T} \int_{0}^{l}|\Delta h(y, t)|^{2} \mathrm{~d} y \mathrm{~d} t .
\end{aligned}
$$


This case requires $(a / l)-\left(\varepsilon_{1} / 2\right) a>0$ and $(a / l)-\left(\varepsilon_{2} / 2\right) a>0$. So, we obtain the following conditions:

$$
\begin{aligned}
& 0<\varepsilon_{1}<\frac{2}{l}, \\
& 0<\varepsilon_{2}<\frac{2}{l} .
\end{aligned}
$$

$$
\begin{aligned}
& \left(\frac{a}{l}-\frac{\varepsilon_{1}}{2} a\right) \int_{0}^{T} \int_{0}^{l}|\Delta u(x, l, t)|^{2} \mathrm{~d} x \mathrm{~d} t \leq \frac{a}{2 \varepsilon_{1}} \int_{0}^{T} \int_{0}^{l}|\Delta h(x, t)|^{2} \mathrm{~d} x \mathrm{~d} t, \\
& \left(\frac{a}{l}-\frac{\varepsilon_{2}}{2} a\right) \int_{0}^{T} \int_{0}^{l}|\Delta u(l, y, t)|^{2} \mathrm{~d} y \mathrm{~d} t \leq \frac{a}{2 \varepsilon_{2}} \int_{0}^{T} \int_{0}^{l}|\Delta h(y, t)|^{2} \mathrm{~d} y \mathrm{~d} t .
\end{aligned}
$$

So, we have

$$
\begin{aligned}
& \int_{0}^{T} \int_{0}^{l}|\Delta u(x, l, t)|^{2} \mathrm{~d} x \mathrm{~d} t \leq \bar{c}_{1} \int_{0}^{T} \int_{0}^{l}|\Delta h(x, t)|^{2} \mathrm{~d} x \mathrm{~d} t, \\
& \int_{0}^{T} \int_{0}^{l}|\Delta u(l, y, t)|^{2} \mathrm{~d} y \mathrm{~d} t \leq \bar{c}_{2} \int_{0}^{T} \int_{0}^{l}|\Delta h(y, t)|^{2} \mathrm{~d} y \mathrm{~d} t,
\end{aligned}
$$

where $\bar{c}_{1}=\left(l / \varepsilon_{1}\left(2-\varepsilon_{1} l\right)\right)$ and $\bar{c}_{2}=\left(l / \varepsilon_{2}\left(2-\varepsilon_{2} l\right)\right)$.

Lemma 2 is been proved.

\subsection{Lipschitz Continuity of the Gradient of Cost Function.} In this section, Lipschitz continuity of the gradient of cost function $J(h)$ is shown, and we also give the Lipschitz constant according to the constant of Lemma 1 and Lemma 2 .

Theorem 1. If the function $\varphi(x, y, t ; h)$ is the solution of the adjoint problem (15), the function $\Delta \varphi(x, y, t)=\varphi(x, y, t ; h+$ $\Delta h)-\varphi(x, y, t ; h)$ is the solution of the following problem:

$$
\begin{aligned}
\Delta \varphi_{t} & =-a\left(\Delta \varphi_{x x}+\Delta \varphi_{y y}\right), \quad(x, y, t) \in \Omega, \\
\Delta \varphi(x, t, T) & =0, \quad x \in[0, l], y \in[0, l], \\
-\left.a \Delta \varphi_{y}(x, y, t)\right|_{y=0} & =0, \\
-\left.a \Delta \varphi_{y}(x, y, t)\right|_{y=l} & =2 \Delta u(x, l, t ; \Delta h), \quad t \in(0, T], x \in[0, l], \\
-\left.a \Delta \varphi_{x}(x, y, t)\right|_{x=0} & =0, \\
-\left.a \Delta \varphi_{x}(x, y, t)\right|_{x=l} & =2 \Delta u(l, y, t ; \Delta h), \quad t \in(0, T], y \in[0, l] .
\end{aligned}
$$

Then, we have the following estimate:

$$
\begin{array}{r}
\left(J^{\prime}(h+\Delta h)-J^{\prime}(h), \Delta h\right) \leq \bar{C}_{1} \int_{0}^{T} \int_{0}^{l}[\Delta h(x, t)]^{2} \mathrm{~d} x \mathrm{~d} t \\
+\bar{C}_{2} \int_{0}^{T} \int_{0}^{l}[\Delta h(y, t)]^{2} \mathrm{~d} y \mathrm{~d} t, \quad \bar{C}_{1}>0, \bar{C}_{2}>0 .
\end{array}
$$

Proof. Considering Lemma 1 and Lemma 2, we multiply both sides of equation (39) by $\Delta u(x, y, t)$ and also simultaneously in time and space integration, and we have

$$
\begin{aligned}
\left(J_{1}^{\prime}(h+\Delta h)-J_{1}^{\prime}(h), \Delta h\right) & =\int_{0}^{T} \int_{0}^{l} \Delta \varphi(l, y, t) \Delta h(y, t) \mathrm{d} y \mathrm{~d} t \\
& =2 \int_{0}^{T} \int_{0}^{l}[\Delta u(l, y, t)]^{2} \mathrm{~d} y \mathrm{~d} t \\
& \leq 2 \bar{c}_{2} \int_{0}^{T} \int_{0}^{l}[\Delta h(y, t)]^{2} \mathrm{~d} y \mathrm{~d} t .
\end{aligned}
$$

Similarly,

$$
\begin{aligned}
\left(J_{2}^{\prime}(h+\Delta h)-J_{2}^{\prime}(h), \Delta h\right) & =\int_{0}^{T} \int_{0}^{l} \Delta \varphi(x, l, t) \Delta h(x, t) \mathrm{d} x \mathrm{~d} t \\
& =2 \int_{0}^{T} \int_{0}^{l}[\Delta u(x, l, t)]^{2} \mathrm{~d} x \mathrm{~d} t \\
& \leq 2 \bar{c}_{1} \int_{0}^{T} \int_{0}^{l}[\Delta h(x, t)]^{2} \mathrm{~d} x \mathrm{~d} t .
\end{aligned}
$$


So, we have

$$
\begin{aligned}
\left(J^{\prime}(h+\Delta h)-J^{\prime}(h), \Delta h\right) & \leq \bar{C}_{1} \int_{0}^{T} \int_{0}^{l}[\Delta h(x, t)]^{2} \mathrm{~d} x \mathrm{~d} t \\
& +\bar{C}_{2} \int_{0}^{T} \int_{0}^{l}[\Delta h(y, t)]^{2} \mathrm{~d} y \mathrm{~d} t
\end{aligned}
$$

where $\bar{C}_{1}=2 \bar{c}_{1}$ and $\bar{C}_{2}=2 \bar{c}_{2}$.

The theorem is been proved.

\section{Sufficient Descent Levenberg-Marquard Algorithm for Solving the Inverse Heat Transfer Problem}

4.1. Sufficient Descent Levenberg-Marquard with Adaptive Parameter (SD-LMAP) Algorithm. Based on the above sections, some gradient methods, such as conjugate gradient method [5], can be used to solve the optimization problem. In practice, the inverse heat transfer problem is ill-posed $[22,23]$, which means that small errors in the measured values of surface temperature can lead to large disturbance in the inverse results. In present, many research works $[16,17,24,25]$ apply the Levenberg-Marquard (L-M) algorithm to solve the inverse heat transfer problem and analyze convergence of a regularizing L-M scheme for nonlinear ill-posed problems. Therefore, this paper considers the L-M algorithm to solve the inverse heat transfer problem.

Because the width and thickness of billet have the same heat flux, the surface temperature on one of it is required to be measured. In order to identify the heat flux $h(\cdot, t)$, we assume that the surface temperature on the $\Gamma_{1}$ can be measured. We have the following cost function:

$$
J_{1}(h)=\frac{1}{2} \int_{0}^{l} \int_{0}^{T}\left\|u_{c}(l, y, t ; h)-u_{e}^{\delta}(l, y, t)\right\|^{2} \mathrm{~d} y \mathrm{~d} t
$$

The iterative process of L-M algorithm is written as

$$
h_{k+1}=h_{k}+\alpha_{k} s_{k}, \quad k=0,1,2, \ldots,
$$

where $\alpha_{k}>0$ is an iteration parameter; in this paper, the exact linear search is used:

$$
J\left(h_{k}-\alpha_{k} s_{k}\right)=\min _{\alpha>0} J\left(h_{k}-\alpha_{k} s_{k}\right),
$$

where $h_{0}$ is a given initial iteration and $s_{k}$ is the search direction determined by L-M algorithm, which is given in the following:

$$
s_{k}=\left(\nabla u_{c}(x, y, t ; h)_{k}^{T} \nabla u_{c}(x, y, t ; h)_{k}+\bar{\mu} I\right)^{-1} d_{k},
$$

where $d_{k}=\left(\nabla J_{1}(h)\right)_{k}$ is the gradient, which decides the iterative direction of $\mathrm{L}-\mathrm{M}$ algorithm, $\bar{\mu}$ is the $\mathrm{L}-\mathrm{M}$ parameter, the adaptive parameter method [19] $\mu_{k}=\left\|J\left(h_{k}\right)\right\|^{2 \delta}$ is used, $\delta$ is the error level, and $I$ is the identity matrix.

Sufficient descent property plays a key role in the global convergence of the iterative method [26]. The sufficient descent condition is defined as

$$
g_{k}^{T} d_{k} \leq-c\left\|g_{k}\right\|^{2}
$$

where $d_{k}$ is the iterative direction at step $k, g_{k}$ is the gradient of cost function at step $k$, and $c$ is a positive constant.

In order to accelerate the convergence rate and reduce the running time, this paper presents a sufficient descent Levenberg-Marquard with adaptive parameter (SD-LMAP) algorithm. The iterative formula is given in the following:

$$
\begin{aligned}
h_{k+1} & =h_{k}+\alpha_{k}\left(\nabla u_{c}(x, y, t ; h)_{k}^{T} \nabla u_{c}(x, y, t ; h)_{k}+\bar{\mu} I\right)^{-1} d_{k}, \\
k & =0,1,2, \ldots,
\end{aligned}
$$

where

$$
d_{k}= \begin{cases}-\left(\nabla J_{1}(h)\right)_{k}, & k=0, \\ -\eta_{k}\left(\nabla J_{1}(h)\right)_{k}+\beta_{k}\left(\nabla J_{1}(h)\right)_{k-1}-\theta_{k} y_{k-1}, & k>0,\end{cases}
$$

with $\quad \eta_{k}=\left(\gamma+\left(\left\|y_{k-1}\right\|^{2} /\left\|\left(\nabla J_{1}(h)\right)_{k}\right\|^{2}\right)\right), \quad \beta_{k}=\left(\left(\nabla J_{1}(h)\right)\right.$ $\left.{ }_{k}^{T} y_{k-1} /\left\|\left(\nabla J_{1}(h)\right)_{k-1}\right\|^{2}\right), \theta_{k}=\left(\left\|\left(\nabla J_{1}(h)\right)_{k}\right\|^{2} /\left\|\left(\nabla J_{1}(h)\right)_{k-1}\right\|^{2}\right)$, and $y_{k}=\left(\nabla J_{1}(h)\right)_{k}-\left(\nabla J_{1}(h)\right)_{k-1}, \gamma \geq 1$.

Theorem 2. If sequences $\left\{h_{k}\right\}$ and $\left\{\bar{s}_{k}\right\}$ are generated by equations (48) and (49), for all $k \geq 0$, the following equation holds:

$$
\left(\nabla J_{1}(h)\right)_{k}^{T} d_{k} \leq-\left\|\left(\nabla J_{1}(h)\right)_{k}\right\|^{2}
$$

Proof. Evidently, it is true for inequality (50) when $k=0$. If $k>0$, multiplying equation (49) by $\left(\nabla J_{1}(h)\right)_{k}^{T}$, we can obtain the following equation: 


$$
\begin{aligned}
& \left(\nabla J_{1}(h)\right)_{k}^{T} d_{k}=-\eta_{k}\left\|\left(\nabla J_{1}(h)\right)_{k}\right\|^{2}+\left(\nabla J_{1}(h)\right)_{k}^{T}\left(\beta_{k}\left(\nabla J_{1}(h)\right)_{k-1}-\theta_{k} y_{k-1}\right) \\
& =-\left(\gamma+\frac{\left\|y_{k-1}\right\|^{2}}{\left\|\left(\nabla J_{1}(h)\right)_{k}\right\|^{2}}\right)\left\|\left(\nabla J_{1}(h)\right)_{k}\right\|^{2} \\
& +\frac{\left(\nabla J_{1}(h)\right)_{k}^{T} y_{k-1}}{\left\|\left(\nabla J_{1}(h)\right)_{k-1}\right\|^{2}}\left(\nabla J_{1}(h)\right)_{k}^{T}\left(\nabla\left(J_{1}(h)\right)_{k-1}\right)-\frac{\left\|\left(\nabla J_{1}(h)\right)_{k}\right\|^{2}}{\left\|\left(\nabla J_{1}(h)\right)_{k-1}\right\|^{2}}\left(\nabla J_{1}(h)\right)_{k}^{T} y_{k-1} \\
& =-\left(\gamma+\frac{\left\|y_{k-1}\right\|^{2}}{\left\|\left(\nabla J_{1}(h)\right)_{k}\right\|^{2}}\right)\left\|\left(\nabla J_{1}(h)\right)_{k}\right\|^{2}+\frac{\left(\nabla J_{1}(h)\right)_{k}^{T} y_{k-1}}{\left\|\left(\nabla J_{1}(h)\right)_{k-1}\right\|^{2}}\left(\nabla J_{1}(h)\right)_{k}^{T}\left[\left(\nabla J_{1}(h)\right)_{k-1}-\left(\nabla J_{1}(h)\right)_{k}\right] \\
& =-\left(\gamma+\frac{\left\|y_{k-1}\right\|^{2}}{\left\|\left(\nabla J_{1}(h)\right)_{k}\right\|^{2}}\right)\left\|\left(\nabla J_{1}(h)\right)_{k}\right\|^{2}-\frac{\left\|\left(\nabla J_{1}(h)\right)_{k}^{T} y_{k-1}\right\|^{2}}{\left\|\left(\nabla J_{1}(h)\right)_{k-1}\right\|^{2}} \\
& \leq-\left(\gamma+\frac{\left\|y_{k-1}\right\|^{2}}{\left\|\left(\nabla J_{1}(h)\right)_{k}\right\|^{2}}\right)\left\|\left(\nabla J_{1}(h)\right)_{k}\right\|^{2} \\
& \leq-\left\|\left(\nabla J_{1}(h)\right)_{k}\right\|^{2} \text {. } \\
& u_{i, j}^{n+1}=A\left(u_{i+1, j}^{n}-2 u_{i, j}^{n}+u_{i-1, j}^{n}\right)+B\left(u_{i, j+1}^{n}-2 u_{i, j}^{n}+u_{i, j-1}^{n}\right) \\
& +Q\left(x_{i}, y_{i}, t_{n}\right) \text {. }
\end{aligned}
$$

Remark 2. The SD-LMAP algorithm is proposed in the framework of LM algorithm because [25, 27] prove the regularizing of LM algorithm. Therefore, the SD-LMAP algorithm also has regularizing. Furthermore, most of the references $[16,28]$ used the LM and the modified LM algorithms to solve the inverse problems in many fields and overcame the ill-posed problem. So, the SD-LMAP algorithm can be used to solve the ill-posed problem.

Remark 3. For the SD-LMAP algorithm, the item $\left(\nabla u_{c}(x, y, t ; h)_{k}^{T} \nabla u_{c}(x, y, t ; h)_{k}+\bar{\mu} I\right)$ has little effect on the iterative direction, but it should be nonsingular. Obviously, this item is not nonsingular when L-M parameter $\bar{\mu}$ is positive. The direction $d_{k}$ of L-M algorithm adopts the gradient scheme, but the sufficient descent direction is used in this paper, and the sufficient descent property of SDLMAP algorithm is analyzed. The SD-LMAP algorithm can guarantee descent at each step and global convergence.

\subsection{Model Discretization and Solving Process of the Inverse Heat Transfer Problem}

4.2.1. Numerical Solution of the Direct Problem. An implementation of the iterative method (SD-LM) requires solving the direct problem effectively in each step. For the numerical solution of direct problem (1)-(6), the discrete model with its boundary conditions is shown in the following based on the finite difference method:
Boundary condition on $\Gamma_{1}(x=l)$ is shown in the following:

$$
\begin{aligned}
u_{i, j}^{n+1}= & 2 A\left(u_{i-1, j}^{n}-u_{i, j}^{n}\right)+B\left(u_{i, j+1}^{n}-2 u_{i, j}^{n}+u_{i, j-1}^{n}\right) \\
& +Q\left(x_{i}, y_{i}, t_{n}\right)-\frac{2}{\rho c \Delta x} q\left(y_{i}, t_{n}\right) .
\end{aligned}
$$

Boundary condition on $\Gamma_{2}(y=l)$ is shown in the following:

$$
\begin{aligned}
u_{i, j}^{n+1}= & A\left(u_{i+1, j}^{n}-2 u_{i, j}^{n}+u_{i-1, j}^{n}\right)+2 B\left(u_{i, j+1}^{n}-u_{i, j}^{n}\right) \\
& +Q\left(x_{i}, y_{i}, t_{n}\right)-\frac{2}{\rho c \Delta y} q\left(x_{i}, t_{n}\right) .
\end{aligned}
$$

Boundary condition on $\Gamma_{3}(x=0)$ is shown in the following:

$$
\begin{aligned}
u_{i, j}^{n+1}= & 2 A\left(u_{i-1, j}^{n}-u_{i, j}^{n}\right)+B\left(u_{i, j+1}^{n}-2 u_{i, j}^{n}+u_{i, j-1}^{n}\right) \\
& +Q\left(x_{i}, y_{i}, t_{n}\right) .
\end{aligned}
$$

Boundary condition on $\Gamma_{4}(y=0)$ is shown in the following

$$
\begin{aligned}
u_{i, j}^{n+1}= & A\left(u_{i+1, j}^{n}-2 u_{i, j}^{n}+u_{i-1, j}^{n}\right)+2 B\left(u_{i, j+1}^{n}-u_{i, j}^{n}\right) \\
& +Q\left(x_{i}, y_{i}, t_{n}\right)
\end{aligned}
$$


where we define $\Delta x=l / M_{1}, i=1,2, \ldots, M_{1}$, $\Delta y=l / M_{2}, j=1,2, \ldots, M_{2}, M_{1}$ is the number of grid at $x$ direction, $M_{2}$ is the number of grid at $y$ direction, $M_{1}$ is equal to $M_{2}$ for the billet, $A=k \Delta t / \rho c \Delta x, B=k \Delta t / \rho c \Delta y$, $\Delta t=t / N$ is time step, $n=1,2, \ldots, N, N$ is the number of time grid. $u_{i, j}^{n}$ is abbreviation of $u\left(x_{i}, y_{j}, t_{n}\right)$, and $u\left(x_{i}, y_{j}, t_{n}\right)$ is the approximate value of $u(x, y, t)$ at the mesh point $\left(x_{i}, y_{i}, t_{n}\right)$.

4.2.2. Solving Process of the Inverse Heat Transfer Problem. We assume that the number of measured point is $M$ on $\Gamma_{1}$ at space. The measured time interval is $\Delta T_{m}$ and the number of measured point is $P=T / \Delta T_{m}$ at space. So, the discrete model of cost function is shown in the following:

$$
J_{1}(h)=\sum_{m=1}^{M} \sum_{p=1}^{P}\left\|u_{c}\left(l, y_{m}, t_{p} ; h\right)-u_{e}^{\delta}\left(l, y_{m}, t_{p}\right)\right\|^{2},
$$

where $u_{e}^{\delta}\left(l, y_{m}, t_{p} ; h\right)$ is the measured value at point $\left(l, y_{m}, t_{p}\right)$ and $u_{c}\left(l, y_{m}, t_{p} ; h\right)$ is the calculated value corresponding to the measured point, which is computed by solving the direct problem (1)-(6). The solving process of the inverse heat transfer problem (7) based on the SD-LM algorithm is given in the following.

Step 1: set $k=1$, given an initial value $h_{0} \in R^{n}$, the maximum iterative number $k_{\max }$, and stopping criteria $\varepsilon_{s p}$

Step 2: compute the temperature $u_{c}\left(l, y_{m}, t_{p} ; h_{k}\right)$ according to equations (53)-(57)

Step 3: calculate the cost function $J_{1}\left(h_{k}\right)$; if $J_{1}\left(h_{k}\right)<\varepsilon_{s p}$ or $k=k_{\max }$, stop; else $k=k+1$, go to Step 4

Step 4: calculate the L-M parameter $\mu_{k}=\left\|J\left(h_{k}\right)\right\|^{2 \delta}$ and the iteration parameter $\alpha_{k}>0$ by equation (46)

Step 5: update the heat flux $h_{k+1}$ according to equation (48), and go to Step 2

\section{Simulation}

In this section, the estimation of heat flux in two-dimensional nonhomogeneous parabolic equation is discussed in two cases. During the process of slab production, the heat transfer coefficient is described by the piecewise linear; thus, the piecewise linear of the heat transfer coefficient is selected in the first case. Furthermore, in order to test the performance of algorithm (SD-LM with adaptive parameter), in the second case, the heat transfer coefficient changing with time is selected. In the next, these two cases and the analyses of simulation results are shown in the following.

Case 1. We consider the process described by means of (1)-(6) for the following values of parameters: $l=3(\mathrm{~m})$, $k=31(\mathrm{~W} /(\mathrm{m} \cdot \mathrm{K})), c=660(\mathrm{~J} /(\mathrm{kg} \cdot \mathrm{K})), \rho=7800\left(\mathrm{~kg} / \mathrm{m}^{3}\right)$, $u_{0}=1000\left({ }^{0} \mathrm{C}\right), u_{w}=30\left({ }^{0} \mathrm{C}\right)$, and $Q(x, y, t)=80 \sin (\pi x)$ $\sin (\pi y) \sin (t)$. Because of $h(y, t)=\bar{\gamma}\left(u(l, y, t)-u_{w}\right)$, if the heat transfer coefficient $\bar{\gamma}$ is known, the heat flux $h(\cdot, t)$ can be obtained. The coefficient $\bar{\gamma}$ is sought in the form given below:

$$
\bar{\gamma}= \begin{cases}\bar{\gamma}_{1}, & 0 \leq x \leq 0.3, \\ \bar{\gamma}_{2}, & 0.3<x \leq 0.7, \\ \bar{\gamma}_{3}, & 0.7<x \leq 1.2, \\ \bar{\gamma}_{4}, & 1.2<x \leq 1.8, \\ \bar{\gamma}_{5}, & 1.8<x \leq 2.4, \\ \bar{\gamma}_{6}, & 2.4<x \leq 3.0,\end{cases}
$$

where the exact values of coefficient $\bar{\gamma}_{i}$ are known and they are equal to $1500\left(\mathrm{~W} /\left(\mathrm{m}^{2} \cdot \mathrm{K}\right)\right), \quad 1000\left(\mathrm{~W} /\left(\mathrm{m}^{2} \cdot \mathrm{K}\right)\right)$, $800\left(\mathrm{~W} /\left(\mathrm{m}^{2} \cdot \mathrm{K}\right)\right), \quad 1800\left(\mathrm{~W} /\left(\mathrm{m}^{2} \cdot \mathrm{K}\right)\right), \quad 1200\left(\mathrm{~W} /\left(\mathrm{m}^{2} \cdot \mathrm{K}\right)\right)$, and $950\left(\mathrm{~W} /\left(\mathrm{m}^{2} \cdot \mathrm{K}\right)\right)$, respectively.

In order to calculate this problem, the finite difference method is used to solve direct heat transfer equation. In executed experiment, we assumed that the values of temperature in six points $\left(N_{m}=6\right)$ are known. The accurate measured values $u_{e}\left(l, y_{m}, t_{p}\right)$ of surface temperature are calculated by the solving the direct heat transfer equation. In order to investigate the stability of numerical solution, the values of measurement temperature were perturbed as

$$
\begin{array}{r}
u_{e}^{\delta}\left(l, y_{m}, t_{p}\right)=u_{e}\left(l, y_{m}, t_{p}\right)+\delta_{e} * r, \\
n * u_{e}\left(l, y_{m}, t_{p}\right),
\end{array}
$$

where $\delta_{e}$ is the error level.

In this simulation experiment, we measure the temperature values $u_{e}\left(l, l / 2, \ldots, t_{p}\right)$ at the point $\left(l, l / 2, t_{p}\right)\left(t_{p}=\right.$ $1, \ldots, P, P=6)$ with the time interval $\Delta T_{m}=20(s)$ and choose the maximum iterative number $N_{\max }=100$. The gradient algorithm (GA), conjugate gradient algorithm (CGA), improved conjugate gradient algorithm (ICGA) [29], LM algorithm [19], sufficient descent Levenberg-Marquard (SD-LM) algorithm, and SD-LMAP are used to estimate the heat transfer coefficient $\bar{\gamma}_{i}, i=1,2, \ldots, 6$. The convergence of the cost function with the iterative number is given in Figure 2. It is can be seen from this figure that, comparing with other methods, the SD-LMAP algorithm obviously accelerates the convergence rate. Furthermore, we choose the stopping criteria $\varepsilon_{s p}=1.0$, and the results of simulation experiment are shown in Table 1. It is can be seen from this table that the iterative number and running time obviously reduced.

In order to investigate the performance of algorithm for overcoming the error, this simulation experiment used the error levels $\delta_{e}=0.0, \delta_{e}=0.01, \delta_{e}=0.03$, and $\delta_{e}=0.05$ to generate the measuring error. The standard deviation $\bar{S}$ is given in the following:

$$
\bar{S}=\frac{1}{n} \sqrt{\sum_{i=1}^{n}\left(\frac{h_{e}^{i}-h_{r}^{i}}{h_{r}^{i}}\right)^{2}}
$$

where $h_{e}^{i}$ is the estimated value of boundary condition, $h_{r}^{i}$ is the true value of boundary condition, and $n$ is the number of sampling point. The inverse results are shown in Table 2. From this table, we can see that the standard deviation obtained by the SD-LMAP algorithm is less than other 

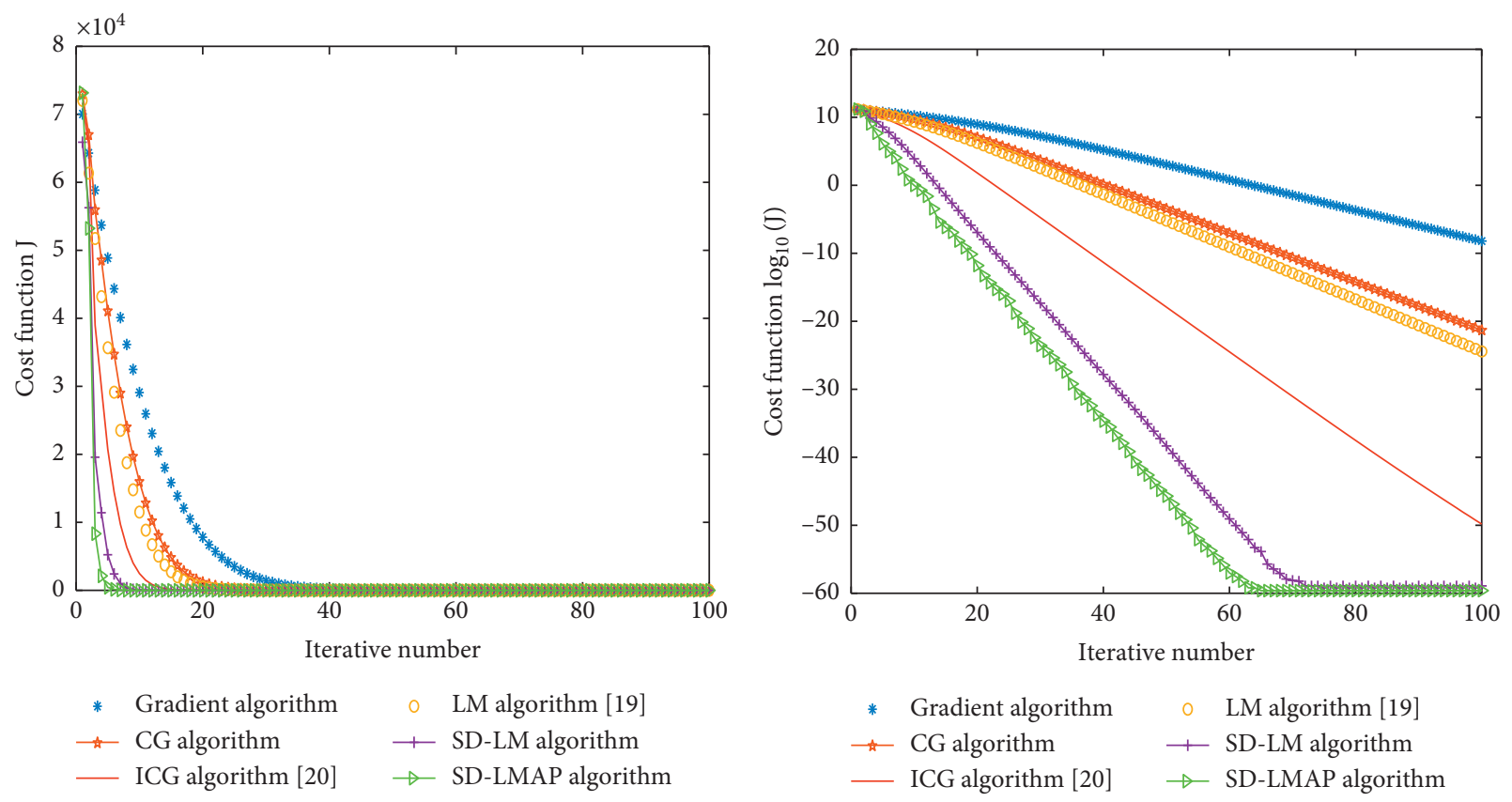

$\begin{array}{llll}* & \text { Gradient algorithm } & \circ & \text { LM algorithm [19] } \\ \text { * } & \text { CG algorithm } & \rightarrow & \text { SD-LM algorithm } \\ & \text { ICG algorithm [20] } & \rightarrow & \text { SD-LMAP algorithm }\end{array}$

(a)

(b)

FIGURE 2: The convergence of the cost function with the number of iterations.

TABLE 1: The comparison of running time and iterative number.

\begin{tabular}{|c|c|c|c|c|c|c|c|c|}
\hline \multirow{3}{*}{ Algorithm } & \multicolumn{8}{|c|}{ Error level } \\
\hline & \multicolumn{2}{|c|}{$\delta_{e}=0.0$} & \multicolumn{2}{|c|}{$\delta_{e}=0.01$} & \multicolumn{2}{|c|}{$\delta_{e}=0.03$} & \multicolumn{2}{|c|}{$\delta_{e}=0.05$} \\
\hline & $\begin{array}{l}\text { Iterative } \\
\text { number }\end{array}$ & $\begin{array}{l}\text { Running } \\
\text { time }\end{array}$ & $\begin{array}{l}\text { Iterative } \\
\text { number }\end{array}$ & $\begin{array}{l}\text { Running } \\
\text { time }\end{array}$ & $\begin{array}{l}\text { Iterative } \\
\text { number }\end{array}$ & $\begin{array}{c}\text { Running } \\
\text { time }\end{array}$ & $\begin{array}{l}\text { Iterative } \\
\text { number }\end{array}$ & Running time \\
\hline GA & 59 & 101.8 & 100 & 273.3 & 100 & 213.4 & 100 & 212.9 \\
\hline CGA & 46 & 63.3 & 52 & 73.3 & 55 & 87.9 & 67 & 151.9 \\
\hline ICGA [29] & 24 & 52.7 & 37 & 54.2 & 41 & 64.12 & 58 & 86.4 \\
\hline LM [19] & 31 & 58.9 & 52 & 119.0 & 54 & 87.9 & 75 & 181.1 \\
\hline SD-LM & 16 & 25.6 & 18 & 39.5 & 15 & 20.3 & 28 & 46.2 \\
\hline SD-LMAP & 6 & 5.81 & 8 & 8.30 & 8 & 7.76 & 10 & 10.05 \\
\hline
\end{tabular}

TABLE 2: The comparison of standard deviation.

\begin{tabular}{|c|c|c|c|c|}
\hline \multirow{2}{*}{ Algorithm } & \multicolumn{4}{|c|}{ Error level } \\
\hline & $\delta_{e}=0.0$ & $\delta_{e}=0.01$ & $\delta_{e}=0.03$ & $\delta_{e}=0.05$ \\
\hline$\overline{\mathrm{GA}}$ & $\bar{S}=0.0123$ & $\bar{S}=0.90$ & $\bar{S}=0.31$ & $\bar{S}=0.36$ \\
\hline CGA & $\bar{S}=0.0074$ & $\bar{S}=0.15$ & $\bar{S}=0.33$ & $\bar{S}=0.39$ \\
\hline ICGA [20] & $\bar{S}=0.0012$ & $\bar{S}=0.11$ & $\bar{S}=0.31$ & $\bar{S}=0.58$ \\
\hline LM [19] & $\bar{S}=0.0018$ & $\bar{S}=0.085$ & $\bar{S}=0.38$ & $\bar{S}=0.41$ \\
\hline SD-LM & $\bar{S}=2.2 \times 10^{-5}$ & $\bar{S}=0.057$ & $\bar{S}=0.26$ & $\bar{S}=0.32$ \\
\hline SD-LMAP & $\bar{S}=1.7561 \times 10^{-15}$ & $\bar{S}=0.0541$ & $\bar{S}=0.1063$ & $\bar{S}=0.2551$ \\
\hline
\end{tabular}

methods, which can eliminate the ill-posedness of inverse problem.

Case 2. In this case, the main parameters and physical parameters are given in Case 1, and the heat flux are changing with time $t$, which is given in the following:

$$
h(t)=1100-1300 * \sin \left(\frac{t}{1000}\right) .
$$

Two hundred points are selected at midsurface as the measured temperature values at positions $\left(l, l / 2, \ldots, t_{p}\right), p=1,2, \ldots, 50$. The SD-LMAP algorithm with other methods is used to estimate the boundary 


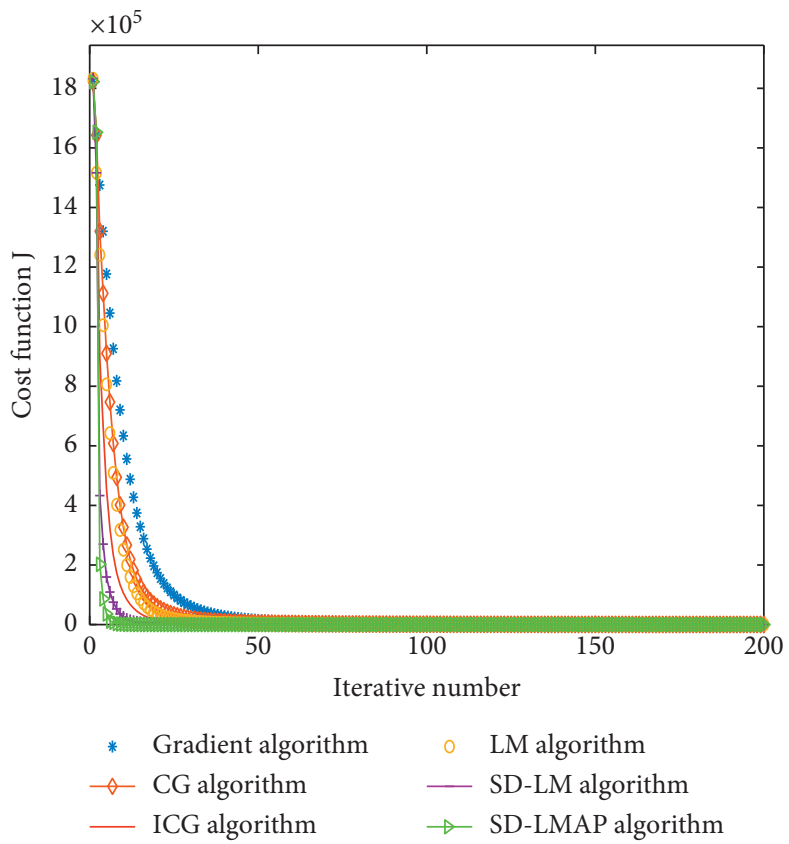

(a)

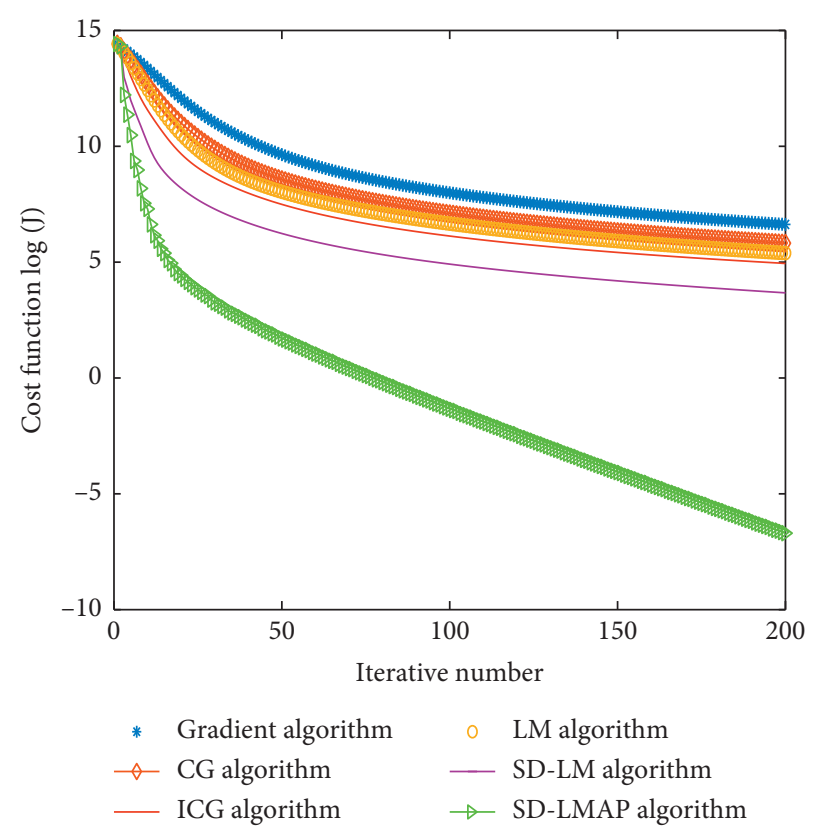

(b)

Figure 3: The convergence of the cost function with the number of iterations.

Table 3: The comparison of the running time and iterative number.

\begin{tabular}{|c|c|c|c|c|c|c|c|c|}
\hline \multirow{3}{*}{ Algorithm } & \multicolumn{8}{|c|}{ Error level } \\
\hline & \multicolumn{2}{|c|}{$\delta_{e}=0.0$} & \multicolumn{2}{|c|}{$\delta_{e}=0.01$} & \multicolumn{2}{|c|}{$\delta_{e}=0.03$} & \multicolumn{2}{|c|}{$\delta_{e}=0.05$} \\
\hline & $\begin{array}{l}\text { Iterative } \\
\text { number }\end{array}$ & $\begin{array}{c}\text { Running } \\
\text { time }\end{array}$ & $\begin{array}{l}\text { Iterative } \\
\text { number }\end{array}$ & $\begin{array}{c}\text { Running } \\
\text { time }\end{array}$ & $\begin{array}{l}\text { Iterative } \\
\text { number }\end{array}$ & $\begin{array}{c}\text { Running } \\
\text { time }\end{array}$ & $\begin{array}{l}\text { Iterative } \\
\text { number }\end{array}$ & Running time \\
\hline GA & 286 & $4.4442 \times 10^{4}$ & 354 & $5.5008 \times 10^{4}$ & 1000 & $1.6247 \times 10^{5}$ & 1000 & $1.6358 \times 10^{5}$ \\
\hline CGA & 180 & $2.8520 \times 10^{4}$ & 223 & $3.3721 \times 10^{4}$ & 865 & $1.3441 \times 10^{5}$ & 1000 & $1.6549 \times 10^{5}$ \\
\hline ICGA [29] & 108 & $1.9320 \times 10^{4}$ & 133 & $2.1007 \times 10^{4}$ & 501 & $7.7851 \times 10^{4}$ & 945 & $1.5479 \times 10^{5}$ \\
\hline LM [19] & 144 & $2.2639 \times 10^{4}$ & 179 & $2.8560 \times 10^{4}$ & 571 & $9.1108 \times 10^{4}$ & 1000 & $1.5956 \times 10^{5}$ \\
\hline SD-LM & 58 & $0.9565 \times 10^{4}$ & 70 & $1.1026 \times 10^{4}$ & 209 & $3.2816 \times 10^{4}$ & 402 & $6.201 \times 10^{4}$ \\
\hline SD-LMAP & 15 & $0.2283 \times 10^{4}$ & 17 & $0.2622 \times 10^{4}$ & 35 & $0.5758 \times 10^{4}$ & 46 & $0.7241 \times 10^{4}$ \\
\hline
\end{tabular}

TABLE 4: The comparison of standard deviation.

\begin{tabular}{|c|c|c|c|c|}
\hline \multirow{2}{*}{ Algorithm } & \multicolumn{4}{|c|}{ Error level } \\
\hline & $\delta_{e}=0.0$ & $\delta_{e}=0.01$ & $\delta_{e}=0.03$ & $\delta_{e}=0.05$ \\
\hline GA & $\bar{S}=0.1289$ & $\bar{S}=0.1420$ & $\bar{S}=0.41$ & $\bar{S}=0.45$ \\
\hline CGA & $\bar{S}=0.0766$ & $\bar{S}=0.0841$ & $\bar{S}=0.343$ & $\bar{S}=0.46$ \\
\hline ICGA [29] & $\bar{S}=0.0322$ & $\bar{S}=0.0415$ & $\bar{S}=0.451$ & $\bar{S}=0.51$ \\
\hline LM [19] & $\bar{S}=0.065$ & $\bar{S}=0.0761$ & $\bar{S}=0.468$ & $\bar{S}=0.47$ \\
\hline SD-LM & $\bar{S}=0.01664$ & $\bar{S}=0.0213$ & $\bar{S}=0.036$ & $\bar{S}=0.087$ \\
\hline SD-LMAP & $\bar{S}=0.0071$ & $\bar{S}=0.0086$ & $\bar{S}=0.0107$ & $\bar{S}=0.021$ \\
\hline
\end{tabular}

condition $h(t)$. In this simulation, the maximum iterative number $k_{\max }$ is 200 and the inverse results are shown in Figure 3. Furthermore, we choose the maximum iterative number $k_{\max }$ is 1000 and the stop criteria $\varepsilon_{s p}$ is 2 , and the performance of SD-LMAP with other methods is listed in Table 3. From Figure 3 and Table 3, it can be seen that the iterative number and running time of SD-LMAP algorithm are obviously reduced.

Furthermore, the measured data with different error levels is used to test the performance of SD-LMAP algorithm, which is generated by equation (60). The SD-LMAP algorithm with other methods are used to estimate the heat 
flux $h(t)$. The results of simulation experiments are shown in Table 4, and it can be seen that the SD-LMAP algorithm eliminates the ill-posedness of this inverse heat transfer problem effectively.

\section{Conclusion}

The two-dimensional nonhomogeneous inverse heat transfer problem is considered. This problem is transformed into the PDE optimization problem. The Lipchitz continuous of the gradient of cost function in the PDE optimization problem with the two-dimensional nonhomogeneous heat transfer equation is proved. Furthermore, a sufficient descent Levenberg-Marquard with adaptive parameter (SDLMAP) algorithm is presented to solve this problem. At last, comparing with other methods, the results of the simulation experiment show that the SD-LMAP algorithm obviously reduces the iterative numbers and running time.

\section{Data Availability}

The analysis result data used to support the findings of this study are included within the article.

\section{Conflicts of Interest}

The authors declare that they have no conflicts of interest.

\section{Acknowledgments}

This work was partly supported by the National Natural Science Foundation of China (61773269), the Natural Science Foundation of Liaoning Province of China (2019-KF03-08), Department of Education of Liaoning Province of China (L201605 and JYT2020016), the Program for Liaoning Excellent Talents in University (LR2019045), the Program for Shenyang High Level Innovative Talents (RC190042), and the Natural Science Foundation of Zhejiang Province of China (LY18F030012).

\section{References}

[1] J. Zhang, D. F. Chen, C. Q. Zhang, S. G. Wang, W. S. Hwang, and M. R. Han, "Effects of an even secondary cooling mode on the temperature and stress fields of round billet continuous casting steel," Journal of Materials Processing Technology, vol. 222, pp. 315-326, 2015.

[2] M. Vynnycky and S. Saleem, "On the explicit resolution of the mushy zone in the modelling of the continuous casting of alloys," Applied Mathematical Modelling, vol. 50, pp. 544-568, 2017.

[3] X. D. Wang, Z. F. Wang, Y. Liu, F. Du, M. Yao, and X. Zhang, "A particle swarm approach for optimization of secondary cooling process in slab continuous casting," International Journal of Heat and Mass Transfer, vol. 93, pp. 250-256, 2016.

[4] J. Yang, Z. Xie, J. Ning, W. Liu, and Z. Ji, "A framework for soft sensing of liquid pool length of continuous casting round blooms," Metallurgical and Materials Transactions B, vol. 45, no. 4, pp. 1545-1556, 2014.

[5] A. Hasanov and B. Pektaş, "Identification of an unknown time-dependent heat source term from overspecified Dirichlet boundary data by conjugate gradient method," Computers \& Mathematics with Applications, vol. 65, no. 1, pp. 42-57, 2013.

[6] B. Pekats and E. Tamci, "The heat flux identification problem for a nonlinear parabolic equation in 2D," Journal of Computational Applied Mathematics, vol. 312, pp. 134-142, 2017.

[7] M. Peixoto, V. Gabriel, L. Q. Ribeiro, C. A. D. Silva, I. A. d. Silva, and V. Seshadri, "Computational and physical simulation of fluid flow inside a beam blank continuous casting mold," Journal of Materials Processing Technology, vol. 233, pp. 89-99, 2016.

[8] S. Rocha and B. Souza, "Modeling and computational simulation of fluid flow, heat transfer and inclusions trajectories in a tundish of a steel continuous casting machine," Journal of Materials Research Technology, vol. 8, pp. 4209-4220, 2019.

[9] S. Chakrabortya, S. Gangulyb, and P. Talukdara, "Determination of optimal taper in continuous casting billet mould using thermo-mechanical models of mould and billet," Journal of Materials Processing Technology, vol. 270, pp. 132-141, 2019.

[10] Z. Wang, S. Qiu, Z. Ruan, and W. Zhang, “A regularized optimization method for identifying the space-dependent source and the initial value simultaneously in a parabolic equation," Computers \& Mathematics with Applications, vol. 67, no. 7, pp. 1345-1357, 2014.

[11] Z. S. Ruan, Z. J. Yang, and X. L. Lu, "An inverse source problem with sparsity constraint for the time-fractional diffusion equation," Advances in Applied Mathematics and Mechanics, vol. 8, no. 1, pp. 1-18, 2016.

[12] W. L. Chen, H. M. Chou, H. L. Lee, and Y. C. Yang, "An inverse hyperbolic heat conduction problem in estimating base heat flux of two-dimensional cylindrical pin fins," International Communications in Heat and Mass Transfer, vol. 52, pp. 90-96, 2014.

[13] Z. X. Zhao and M. K. Banda, "Simultaneous identification of diffusion coefficient, spacewise dependent source and initial value for one-dimensional heat equation," Mathematical Methods in the Applied Science, vol. 40, pp. 3405-3832, 2016.

[14] J. V. Beck and K. A. Woodbury, "Inverse heat conduction problem: sensitivity coefficient insights, filter coefficients, and intrinsic verification," International Journal of Heat and Mass Transfer, vol. 97, pp. 578-588, 2016.

[15] K. H. Lee, "Application of repulsive particle swarm optimization for inverse heat conduction problem-parameter estimations of unknown plane heat source," International Journal of Heat and Mass Transfer, vol. 137, pp. 268-279, 2019.

[16] M. Cui, K. Yang, X. L. Xu, S. D. Wang, and X. W. Gao, “A modified Levenberg-Marquardt algorithm for simultaneous estimation of multi-parameters of boundary heat flux by solving transient nonlinear inverse heat conduction problems," International Journal of Heat and Mass Transfer, vol. 97, pp. 908-916, 2016.

[17] M. Cui, Y. Zhao, B. Xu, and X. W. Gao, “A new approach for determining damping factors in Levenberg-Marquardt algorithm for solving an inverse heat conduction problem," International Journal of Heat and Mass Transfer, vol. 107, pp. 747-754, 2017.

[18] E. P. Oliveira, G. D. M. Stieven, E. F. Lins, and J. R. P. Vaz, “An inverse approach for the interfacial heat transfer parameters in alloys solidification," Applied Thermal Engineering, vol. 155, pp. 365-372, 2019.

[19] Y. Yu and X. C. Luo, "Estimation of heat transfer coefficients and heat flux on the billet surface by an integrated approach," International Journal of Heat and Mass Transfer, vol. 90, pp. 645-653, 2015. 
[20] Y. Wang, X. C. Luo, Y. Yu, and Q. Yin, "Evaluation of heat transfer coefficients in continuous casting under large disturbance by weighted least squares levenberg-marquardt method," Applied Thermal Engineering, vol. 111, pp. 989-996, 2017.

[21] O. A. Ladyzhenskaya, Boundary Value Probelms in Mathematical Physics, Springer-Verlag, New York, NY, USA, 1985.

[22] D. W. Marquardt, "An algorithm for least-squares estimation of nonlinear parameters," Journal of the Society for Industrial and Applied Mathematics, vol. 11, no. 2, pp. 431-441, 1963.

[23] L. C. Brandao, R. T. Coelho, and C. H. Lauro, "Contribution to dynamic characteristics of the cutting temperature in the drilling process considering one dimension heat flow," Applied Thermal Engineering, vol. 31, no. 17-18, pp. 3806-3813, 2011.

[24] M. A. Iglesias and C. Dawson, "The regularizing levenbergmarquardt scheme for history matching of petroleum reservoirs," Computational Geosciences, vol. 17, no. 6, pp. 1033-1053, 2013.

[25] M. Hochbruck and M. Hönig, "On the convergence of a regularizing levenberg-marquardt scheme for nonlinear illposed problems," Numerische Mathematik, vol. 115, no. 1, pp. 71-79, 2010.

[26] G. Yuan, X. Lu, and Z. Wei, "A conjugate gradient method with descent direction for unconstrained optimization," Journal of Computational and Applied Mathematics, vol. 233, no. 2, pp. 519-530, 2009.

[27] C. Böckmann, A. Kammanee, and A. Braun, "Logarithmic convergence rate of levenberg-marquardt method with application to an inverse potential problem," Journal of Inverse and III-Posed Problems, vol. 19, pp. 345-367, 2011.

[28] A. Kleefeld and M. Rei, el, The levenberg-marquardt method applied to a parameter estimation problem arising from electrical resistivity tomography," Applied Mathematics and Computation, vol. 217, no. 9, pp. 4490-4501, 2011.

[29] Y. Wang, X. C. Luo, F. Zhang, and S. Wang, "GPU-based model predictive control for continuous casting spray cooling control system using particle swarm optimization," Control Engineering Practice, vol. 84, pp. 349-364, 2019. 